Pure and Applied Mathematics Quarterly

Volume 3, Number 2

(Special Issue: In honor of

Leon Simon, Part 1 of 2)

$499-512,2007$

\title{
A Short Proof for the Convergence of the Yamabe Flow on $S^{n}$
}

\author{
Simon Brendle
}

\section{INTRODUCTION}

Let $M$ be a Riemannian manifold of dimension $n \geq 3$ with Riemannian metric $g$. Consider the following evolution equation for the Riemannian metric:

$$
\frac{\partial g}{\partial t}=-\left(R_{g}-r_{g}\right) g \text {. }
$$

Here, $R_{g}$ denotes the scalar curvature associated with the metric $g$ and

$$
r_{g}=\frac{\int_{M} R_{g} d v o l_{g}}{\int_{M} d v o l_{g}}
$$

is the mean value of the scalar curvature on $M$. The evolution equation (1) is known as the Yamabe flow. It is well known that (1) can be reduced to a nonlinear partial differential equation of parabolic type. To see this, we choose a background metric $g_{0}$ which is conformal to the initial metric. Since the Yamabe flow preserves the conformal structure, the metric $g$ can be written in the form $g=u^{\frac{4}{n-2}} g_{0}$, where $u$ is a positive function. Then the Yamabe flow is equivalent to the equation

$$
\frac{\partial}{\partial t} u^{\frac{n+2}{n-2}}=\frac{n+2}{4}\left(\frac{4(n-1)}{n-2} \Delta_{g_{0}} u-R_{g_{0}} u+r_{g} u^{\frac{n+2}{n-2}}\right),
$$

where $R_{g_{0}}$ denotes the scalar curvature of the background metric $g_{0}$ and $\Delta_{g_{0}}$ is the Laplace operator associated with $g_{0}$.

The Yamabe flow was proposed by Richard Hamilton in the 1980s as a tool for constructing metrics of constant scalar curvature in a given conformal class [7].

Received November 10, 2005. 
Hamilton proved that the Yamabe flow has a global solution for every initial metric. The asymptotic behavior of the Yamabe flow was first studied by Bennett Chow [5]. Chow proved that the flow approaches a metric of constant scalar curvature provided that the initial metric is locally conformally flat and has positive Ricci curvature.

Rugang Ye proved the convergence of the Yamabe flow assuming only that the initial metric is locally conformally flat [14]. Ye's proof is based on an estimate for the gradient of the function $\log u$ which is established using the method of moving planes.

A different approach was developed by Hartmut Schwetlick and Michael Struwe [11]. Schwetlick and Struwe established new integral estimates for the scalar curvature, which allowed them to perform a blow-up analysis. Moreover, they were able to rule out the formation of a singularity provided that the dimension is less than 6 and the initial energy is below a certain threshold. Unlike the method of moving planes, the techniques used in [11] are not limited to the locally conformally flat case.

In a previous paper, it was shown that the condition $n<6$ suffices to rule out the formation of a singularity as $t \rightarrow \infty$ [4]. The proof of this result uses the positive mass theorem. This is perhaps not surprising, as the positive mass theorem plays a key role in Richard Schoen's solution of the Yamabe problem (see $[8],[10])$. Another tool used in [4] is the Lojasiewicz inequality. The use of this tool in geometric analysis was pioneered by Leon Simon in an influential paper on the asymptotic behavior of gradient flows [12].

The purpose of this note is to provide a short proof for the convergence of the Yamabe flow on $S^{n}$. This is a special case of Ye's theorem [14]. The proof given in this paper is based on variational techniques, and does not use the method of moving planes.

Theorem. Consider an initial metric on $S^{n}$ which is conformally equivalent to the standard metric. Then the Yamabe flow has a global solution, which converges exponentially to a metric of constant sectional curvature.

\section{The YAmaBe FLOW ON $S^{n}$}

Throughout this paper, we assume that the initial metric is conformally equivalent to the standard metric $g_{S^{n}}$ on $S^{n}$. It follows from a result of Hamilton that the Yamabe flow has a global solution $\{g(t): t \geq 0\}$. Moreover, the solution 
satisfies

$$
\lim _{t \rightarrow \infty} \int_{S^{n}}\left|R_{g(t)}-r_{g(t)}\right|^{s} d v o l_{g(t)}=0
$$

for $s<\frac{n+2}{2}$. If the initial metric has positive scalar curvature, this follows from a result of Schwetlick and Struwe (see [11], Lemma 3.3). For the general case, see [4], Proposition 3.1.

The time-dependent metric $g(t)$ can be written in the form $g(t)=u(t)^{\frac{4}{n-2}} g_{S^{n}}$ for some positive function $u(t)$. Without loss of generality, we may assume that the volume is normalized to 1 . This implies

$$
\int_{S^{n}} u(t)^{\frac{2 n}{n-2}} d v o l_{S^{n}}=1
$$

for all $t \geq 0$. For abbreviation, let

$$
r_{\infty}=\lim _{t \rightarrow \infty} r_{g(t)}
$$

Since the function $t \mapsto r_{g(t)}$ is decreasing, the limit always exists.

For each point $p \in B^{n+1}$, we define a function $\delta_{p}: S^{n} \rightarrow \mathbb{R}$ by

$$
\delta_{p}(x)=\left(\frac{n(n-1)}{r_{\infty}}\right)^{\frac{n-2}{4}}\left(\frac{1-|p|^{2}}{1+2\langle p, x\rangle+|p|^{2}}\right)^{\frac{n-2}{2}}
$$

for $x \in S^{n}$. The conformal metric $\delta_{p}^{\frac{4}{n-2}} g_{S^{n}}$ has constant scalar curvature $r_{\infty}$. Therefore, the function $\delta_{p}$ satisfies the nonlinear elliptic partial differential equation

$$
\frac{4(n-1)}{n-2} \Delta_{S^{n}} \delta_{p}-n(n-1) \delta_{p}+r_{\infty} \delta_{p}^{\frac{n+2}{n-2}}=0 .
$$

For later use, we define a function $\xi_{p}: S^{n} \rightarrow \mathbb{R}^{n+1}$ by

$$
\xi_{p}(x)=\delta_{p}(x)\left[p+\frac{1-|p|^{2}}{1+2\langle p, x\rangle+|p|^{2}}(x+p)\right] \in \mathbb{R}^{n+1}
$$

for $x \in S^{n}$. For every point $x \in S^{n}$, we have

$$
\frac{\partial}{\partial p} \delta_{p}(x)=-\frac{n-2}{1-|p|^{2}} \xi_{p}(x) \in \mathbb{R}^{n=1}
$$

Hence, the function $\xi_{p}$ is a solution of the linearized equation.

$$
\frac{4(n-1)}{n-2} \Delta_{S^{n}} \xi_{p}-n(n-1) \xi_{p}+\frac{n+2}{n-2} r_{\infty} \delta_{p}^{\frac{4}{n-2}} \xi_{p}=0 .
$$

Our goal is to show that the Yamabe flow approaches a metric of constant scalar curvature as $t \rightarrow \infty$. To this end, we consider a sequence of times $\left\{t_{\nu}: \nu \in \mathbb{N}\right\}$ 
such that $t_{\nu} \rightarrow \infty$ as $\nu \rightarrow \infty$. For abbreviation, let $u_{\nu}=u\left(t_{\nu}\right)$ for all $\nu \in \mathbb{N}$.

Lemma 1. After passing to a subsequence if necessary, we can find a positive integer $m$ and a family of points $\left\{p_{k, \nu}: 1 \leq k \leq m, \nu \in \mathbb{N}\right\} \subset B^{n+1}$ with the following properties:

(i) We have

$$
\left\|u_{\nu}-\sum_{k=1}^{m} \delta_{p_{k, \nu}}\right\|_{H^{1}\left(S^{n}\right)} \rightarrow 0
$$

as $\nu \rightarrow \infty$.

(ii) For every pair $i \neq j$ we have

$$
\frac{\left|p_{i, \nu}-p_{j, \nu}\right|^{2}}{\left(1-\left|p_{i, \nu}\right|^{2}\right)\left(1-\left|p_{j, \nu}\right|^{2}\right)} \rightarrow \infty
$$

as $\nu \rightarrow \infty$.

(iii) The integer $m$ is given by

$$
r_{\infty}=\left(m Y\left(S^{n}\right)^{\frac{n}{2}}\right)^{\frac{2}{n}}
$$

Proof. Using (4) with $\gamma=\frac{2 n}{n+2}$, we obtain

$$
\int_{S^{n}}\left|\frac{4(n-1)}{n-2} \Delta_{S^{n}} u_{\nu}-n(n-1) u_{\nu}+r_{\infty} u_{\nu}^{\frac{n+2}{n-2}}\right|^{\frac{2 n}{n+2}} d v o l_{S^{n}} \rightarrow 0
$$

as $\nu \rightarrow \infty$. Hence, we may apply a general compactness result due to Michael Struwe [13]. After passing to a subsequence if necessary, we obtain

$$
\begin{aligned}
\| u_{\nu}-u_{\infty}-\sum_{k=1}^{l}\left(\frac{n(n-1)}{r_{\infty}}\right)^{\frac{n-2}{4}} & \\
& \cdot\left(\frac{2 \varepsilon_{k, \nu}}{\varepsilon_{k, \nu}^{2}+\left(1-\frac{1}{4} \varepsilon_{k, \nu}^{2}\right)\left|x-a_{k, \nu}\right|^{2}}\right)^{\frac{n-2}{2}} \|_{H^{1}\left(S^{n}\right)} \rightarrow 0
\end{aligned}
$$

as $\nu \rightarrow \infty$. Here, $l$ is a non-negative integer, and $\left\{a_{k, \nu}: 1 \leq k \leq l, \nu \in \mathbb{N}\right\}$ is a family of points on $S^{n}$. Moreover, $\left\{\varepsilon_{k, \nu}: 1 \leq k \leq l, \nu \in \mathbb{N}\right\}$ is a family of positive real numbers such that $\varepsilon_{k, \nu} \rightarrow 0$ as $\nu \rightarrow \infty$. For every pair $i \neq j$, we have

$$
\frac{\left(\varepsilon_{i, \nu}-\varepsilon_{j, \nu}\right)^{2}+\left|a_{i, \nu}-a_{j, \nu}\right|^{2}}{\varepsilon_{i, \nu} \varepsilon_{j, \nu}} \rightarrow \infty
$$


as $\nu \rightarrow \infty$. Finally, the function $u_{\infty}$ is a non-negative smooth solution of the partial differential equation

$$
\frac{4(n-1)}{n-2} \Delta_{S^{n}} u_{\infty}-n(n-1) u_{\infty}+r_{\infty} u_{\infty}^{\frac{n+2}{n-2}}=0 .
$$

Using a theorem of Obata, we conclude that either $u_{\infty}=0$ or $u_{\infty}=\delta_{p}$ for some point $p \in B^{n+1}$ (see [9], Proposition 6.1). Hence, we need to distinguish two cases:

Case 1: Suppose that $u_{\infty}=0$. In this case, we put $m=l$, and

$$
p_{k, \nu}=-\frac{2-\varepsilon_{k, \nu}}{2+\varepsilon_{k, \nu}} a_{k, \nu}
$$

for $1 \leq k \leq l$.

Case 2: Suppose that $u_{\infty}=\delta_{p}$ for some $p \in B^{n+1}$. In this case, we put $m=l+1$. Moreover, we define

$$
p_{k, \nu}=\left\{\begin{array}{lr}
-\frac{2-\varepsilon_{k, \nu}}{2+\varepsilon_{k, \nu}} a_{k, \nu} & \text { for } 1 \leq k \leq l \\
p & \text { for } k=l+1
\end{array} .\right.
$$

The assertion follows now from (14) and (15).

The condition (12) has an interesting interpretation in terms of hyperbolic geometry. Note that

$$
\left(\sinh \frac{d_{h y p}\left(p_{i, \nu}, p_{j, \nu}\right)}{2}\right)^{2}=\frac{\left|p_{i, \nu}-p_{j, \nu}\right|^{2}}{\left(1-\left|p_{i, \nu}\right|^{2}\right)\left(1-\left|p_{j, \nu}\right|^{2}\right)},
$$

where $d_{\text {hyp }}(\cdot, \cdot)$ denotes the geodesic distance of two points in $B^{n+1}$ relative to the hyperbolic metric on $B^{n+1}$. (This formula is a consequence of Corollary A.5.8 in [3].) Therefore, (12) is equivalent to $d_{h y p}\left(p_{i, \nu}, p_{j, \nu}\right) \rightarrow \infty$ as $\nu \rightarrow \infty$.

For each $\nu \in \mathbb{N}$, we minimize the expression

$$
\begin{aligned}
\int_{S^{n}}\left[\frac{4(n-1)}{n-2}\left|d\left(u_{\nu}-\sum_{k=1}^{m} \alpha_{k} \delta_{q_{k}}\right)\right|_{S^{n}}^{2}\right. & \\
+ & \left.n(n-1)\left(u_{\nu}-\sum_{k=1}^{m} \alpha_{k} \delta_{q_{k}}\right)^{2}\right] d v o l_{S^{n}}
\end{aligned}
$$

subject to the constaints

$$
\frac{1}{2} \leq \alpha_{k} \leq 2
$$


and

$$
\left|q_{k}-p_{k, \nu}\right| \leq \frac{1}{2}\left(1-\left|p_{k, \nu}\right|\right)
$$

for $1 \leq k \leq m$.

Proposition 2. Suppose that, for each $\nu \in \mathbb{N},\left\{\left(\alpha_{k, \nu}, q_{k, \nu}\right): 1 \leq k \leq m\right\}$ is an $m$-tuplet which minimizes the expression (16) subject to the constraints (17),(18). Then the following statements hold:

(i) We have

$$
\left\|u_{\nu}-\sum_{k=1}^{m} \alpha_{k, \nu} \delta_{q_{k, \nu}}\right\|_{H^{1}\left(S^{n}\right)} \rightarrow 0
$$

as $\nu \rightarrow \infty$.

(ii) For every pair $i \neq j$ we have

as $\nu \rightarrow \infty$.

$$
\frac{\left|q_{i, \nu}-q_{j, \nu}\right|^{2}}{\left(1-\left|q_{i, \nu}\right|^{2}\right)\left(1-\left|q_{j, \nu}\right|^{2}\right)} \rightarrow \infty
$$

(iii) For every $1 \leq k \leq m$, we have

$$
\alpha_{k, \nu} \rightarrow 1
$$

and

$$
\frac{\left|q_{k, \nu}-p_{k, \nu}\right|}{1-\left|p_{k, \nu}\right|} \rightarrow 0
$$

as $\nu \rightarrow \infty$.

Proof. Since the expression (16) attains its minimum at $\left\{\left(\alpha_{k, \nu}, q_{k, \nu}\right): 1 \leq k \leq\right.$ $m\}$, the estimate (19) is an immediate consequence of (11). Similarly, (20) follows from (12). To see this, consider a pair $i \neq j$. Using the triangle inequality, we obtain

$$
\left|p_{i, \nu}-p_{j, \nu}\right| \leq\left|q_{i, \nu}-q_{j, \nu}\right|+\left(1-\left|q_{i, \nu}\right|\right)+\left(1-\left|q_{j, \nu}\right|\right),
$$

hence

$$
\begin{aligned}
& \left|p_{i, \nu}-p_{j, \nu}\right|^{2} \\
& \leq 3\left|q_{i, \nu}-q_{j, \nu}\right|^{2}+3\left(1-\left|q_{i, \nu}\right|\right)^{2}+3\left(1-\left|q_{j, \nu}\right|\right)^{2} \\
& =3\left|q_{i, \nu}-q_{j, \nu}\right|^{2}+3\left(\left|q_{i, \nu}\right|-\left|q_{j, \nu}\right|\right)^{2}+6\left(1-\left|q_{i, \nu}\right|\right)\left(1-\left|q_{j, \nu}\right|\right) \\
& \leq 6\left|q_{i, \nu}-q_{j, \nu}\right|^{2}+6\left(1-\left|q_{i, \nu}\right|\right)\left(1-\left|q_{j, \nu}\right|\right)
\end{aligned}
$$


Moreover, we have

$$
\begin{aligned}
\left(1-\left|p_{i, \nu}\right|^{2}\right)\left(1-\left|p_{j, \nu}\right|^{2}\right) & \geq\left(1-\left|p_{i, \nu}\right|\right)\left(1-\left|p_{j, \nu}\right|\right) \\
& \geq \frac{4}{9}\left(1-\left|q_{i, \nu}\right|\right)\left(1-\left|q_{j, \nu}\right|\right) \\
& \geq \frac{1}{9}\left(1-\left|q_{i, \nu}\right|^{2}\right)\left(1-\left|q_{j, \nu}\right|^{2}\right) .
\end{aligned}
$$

Thus, we conclude that

$$
\frac{\left|p_{i, \nu}-p_{j, \nu}\right|^{2}}{\left(1-\left|p_{i, \nu}\right|^{2}\right)\left(1-\left|p_{j, \nu}\right|^{2}\right)} \leq 54 \frac{\left|q_{i, \nu}-q_{j, \nu}\right|^{2}}{\left(1-\left|q_{i, \nu}\right|^{2}\right)\left(1-\left|q_{j, \nu}\right|^{2}\right)}+\frac{27}{2}
$$

Hence, it follows from Lemma 1 that

$$
\frac{\left|q_{i, \nu}-q_{j, \nu}\right|^{2}}{\left(1-\left|q_{i, \nu}\right|^{2}\right)\left(1-\left|q_{j, \nu}\right|^{2}\right)} \rightarrow \infty
$$

as $\nu \rightarrow \infty$. This completes the proof of (20). Finally, it follows from (11) and (19) that

$$
\left\|\sum_{k=1}^{m} \alpha_{k, \nu} \delta_{q_{k, \nu}}-\sum_{k=1}^{m} \delta_{p_{k, \nu}}\right\|_{H^{1}\left(S^{n}\right)} \rightarrow 0
$$

as $\nu \rightarrow \infty$. From this, the relations (21) and (22) follow easily.

For each $\nu \in \mathbb{N}$, we define a function $v_{\nu}$ by

$$
v_{\nu}=\sum_{k=1}^{m} \alpha_{k, \nu} \delta_{q_{k, \nu}},
$$

where $\left\{\left(\alpha_{k, \nu}, q_{k, \nu}\right): 1 \leq k \leq m\right\}$ is chosen so as to minimize the expression (16). Moreover, we define a function $w_{\nu}$ by

$$
w_{\nu}=u_{\nu}-\sum_{k=1}^{m} \alpha_{k, \nu} \delta_{q_{k, \nu}}
$$

Clearly, $\left\|w_{\nu}\right\|_{H^{1}\left(S^{n}\right)} \rightarrow 0$ as $\nu \rightarrow \infty$.

We next derive an upper bound for the Yamabe energy of the function $v_{\nu}$. This estimate plays a key role in our analysis.

Proposition 3. The function $v_{\nu}$ satisfies the estimate

$$
\begin{aligned}
& \int_{S^{n}}\left(\frac{4(n-1)}{n-2}\left|d v_{\nu}\right|_{S^{n}}^{2}+n(n-1) v_{\nu}^{2}\right) d v o l_{S^{n}} \\
& \leq r_{\infty}\left(\int_{S^{n}} v_{\nu}^{\frac{2 n}{n-2}} d v o l_{S^{n}}\right)^{\frac{n-2}{n}}
\end{aligned}
$$


for all $\nu \in \mathbb{N}$.

Proof. Note that

$$
\int_{S^{n}} \sum_{k=1}^{m} \delta_{q_{k, \nu}}^{\frac{2 n}{n-2}} d v o l_{S^{n}}=m\left(\frac{Y\left(S^{n}\right)}{r_{\infty}}\right)^{\frac{n}{2}}=1 .
$$

Using (8) and Hölder's inequality, we obtain

$$
\begin{aligned}
& \int_{S^{n}}\left(\frac{4(n-1)}{n-2}\left|d v_{\nu}\right|_{S^{n}}^{2}+n(n-1) v_{\nu}^{2}\right) d v o l_{S^{n}} \\
& =\int_{S^{n}} \sum_{i, j=1}^{m} \alpha_{i, \nu} \alpha_{j, \nu}\left(\frac{4(n-1)}{n-2}\left\langle d \delta_{q_{i, \nu}}, d \delta_{q_{j, \nu}}\right\rangle_{S^{n}}+n(n-1) \delta_{q_{i, \nu}} \delta_{q_{j, \nu}}\right) d v o l_{S^{n}} \\
& =r_{\infty} \int_{S^{n}} \sum_{i, j=1}^{m} \alpha_{i, \nu} \alpha_{j, \nu} \delta_{q_{i, \nu}}^{\frac{n+2}{n-2}} \delta_{q_{j, \nu}} d v o l_{S^{n}} \\
& \leq r_{\infty} \int_{S^{n}}\left(\sum_{k=1}^{m} \delta_{q_{k, \nu}}^{\frac{2 n}{n-2}}\right)^{\frac{2}{n}} v_{\nu}^{2} d v o l_{S^{n}} \\
& \leq r_{\infty}\left(\int_{S^{n}} \sum_{k=1}^{m} \delta_{q_{k, \nu}}^{\frac{2 n}{n-2}} d v o l_{S^{n}}\right)^{\frac{2}{n}}\left(\int_{S^{n}} v_{\nu}^{\frac{2 n}{n-2}} d v o l_{S^{n}}\right)^{\frac{n-2}{n}} \\
& =r_{\infty}\left(\int_{S^{n}} v_{\nu}^{\frac{2 n}{n-2}} d v o l_{S^{n}}\right)^{\frac{n-2}{n}} \cdot
\end{aligned}
$$

This completes the proof.

Corollary 4. The function $v_{\nu}$ satisfies

$$
\begin{aligned}
& \int_{S^{n}}\left(\frac{4(n-1)}{n-2}\left|d v_{\nu}\right|_{S^{n}}^{2}+n(n-1) v_{\nu}^{2}\right) d \operatorname{vol}_{S^{n}} \\
& \leq \frac{2}{n} r_{\infty}+\frac{n-2}{n} r_{\infty} \int_{S^{n}} v_{\nu}^{\frac{2 n}{n-2}} d \operatorname{vol}_{S^{n}}
\end{aligned}
$$

Proof. Since the function $x \mapsto x^{\frac{n-2}{n}}$ is concave, we have

$$
x^{\frac{n-2}{n}} \leq \frac{2}{n}+\frac{n-2}{n} x
$$

for all $x \geq 0$. Hence, the assertion follows from Proposition 3 .

Besides Corollary 4, we shall need an estimate for the second variation operator of the Yamabe functional at $v_{\nu}$. Since this estimate is standard, we present only a brief sketch of the proof. 
Proposition 5. There exists a positive constant $\eta$ such that

$$
\begin{aligned}
& \frac{n+2}{n-2} r_{\infty} \int_{S^{n}} v_{\nu}^{\frac{4}{n-2}} w_{\nu}^{2} d v o l_{S^{n}} \\
& \leq(1-\eta) \int_{S^{n}}\left(\frac{4(n-1)}{n-2}\left|d w_{\nu}\right|_{S^{n}}^{2}+n(n-1) w_{\nu}^{2}\right) d v o l_{S^{n}}
\end{aligned}
$$

if $\nu$ is sufficiently large.

Proof. Recall that the expression (16) attains its minimum at $\left\{\left(\alpha_{k, \nu}, q_{k, \nu}\right): 1 \leq\right.$ $k \leq m\}$. It follows from (21) and (22) that none of the constraints (17) and (18) is binding if $\nu$ is sufficiently large. The first order conditions for a minimum are

$$
\begin{aligned}
0 & =\int_{S^{n}}\left(\frac{4(n-1)}{n-2} \Delta_{S^{n}} \delta_{q_{k, \nu}}-n(n-1) \delta_{q_{k, \nu}}\right) w_{\nu} d v o l_{S^{n}} \\
& =-r_{\infty} \int_{S^{n}} \delta_{q_{k, \nu}}^{\frac{n+2}{n-2}} w_{\nu} d v o l_{S^{n}}
\end{aligned}
$$

and

$$
\begin{aligned}
0 & =\int_{S^{n}}\left(\frac{4(n-1)}{n-2} \Delta_{S^{n}} \xi_{q_{k, \nu}}-n(n-1) \xi_{q_{k, \nu}}\right) w_{\nu} \operatorname{dvol}_{S^{n}} \\
& =-\frac{n+2}{n-2} r_{\infty} \int_{S^{n}} \delta_{q_{k, \nu}}^{\frac{4}{n-2}} \xi_{q_{k, \nu}} w_{\nu} d v o l_{S^{n}}
\end{aligned}
$$

for $1 \leq k \leq m$. The assertion follows now from standard arguments. A proof for $n=3$ can be found in [2], Lemma A2. The general case is discussed in [1].

Proposition 6. We have

$$
\begin{aligned}
& \int_{S^{n}}\left(\frac{4(n-1)}{n-2}\left|d u_{\nu}\right|_{S^{n}}^{2}+n(n-1) u_{\nu}^{2}\right) d v o l_{S^{n}} \\
& \leq r_{\infty}+\frac{2}{\eta Y\left(S^{n}\right)}\left(\int_{S^{n}} \mid \frac{4(n-1)}{n-2} \Delta_{S^{n}} u_{\nu}\right. \\
& \left.\quad-n(n-1) u_{\nu}+\left.r_{\infty} u_{\nu}^{\frac{n+2}{n-2}}\right|^{\frac{2 n}{n+2}} d v o l_{S^{n}}\right)^{\frac{n+2}{n}}
\end{aligned}
$$

if $\nu$ is sufficiently large.

Proof. Using the identity

$$
\int_{S^{n}} u_{\nu}^{\frac{2 n}{n-2}} d v^{n o l} S_{S^{n}}=1
$$


and the relation $u_{\nu}=v_{\nu}+w_{\nu}$, we obtain

$$
\begin{aligned}
& \int_{S^{n}}\left(\frac{4(n-1)}{n-2}\left|d u_{\nu}\right|_{S^{n}}^{2}+n(n-1) u_{\nu}^{2}\right) d v o l_{S^{n}} \\
& =\frac{n-2}{n} r_{\infty}-\frac{n-2}{n} r_{\infty} \int_{S^{n}} v_{\nu}^{\frac{2 n}{n-2}} d v o l_{S^{n}} \\
& +\int_{S^{n}}\left(\frac{4(n-1)}{n-2}\left|d v_{\nu}\right|_{S^{n}}^{2}+n(n-1) v_{\nu}^{2}\right) d v o l_{S^{n}} \\
& -2 \int_{S^{n}}\left(\frac{4(n-1)}{n-2} \Delta_{S^{n}} u_{\nu}-n(n-1) u_{\nu}+r_{\infty} u_{\nu}^{\frac{n+2}{n-2}}\right) w_{\nu} d v o l_{S^{n}} \\
& -\int_{S^{n}}\left(\frac{4(n-1)}{n-2}\left|d w_{\nu}\right|_{S^{n}}^{2}+n(n-1) w_{\nu}^{2}-\frac{n+2}{n-2} r_{\infty} v_{\nu}^{\frac{4}{n-2}} w_{\nu}^{2}\right) d v o l_{S^{n}} \\
& +r_{\infty} \int_{S^{n}}\left(\frac{n-2}{n} v_{\nu}^{\frac{2 n}{n-2}}-\frac{n+2}{n-2} v_{\nu}^{\frac{4}{n-2}} w_{\nu}^{2}\right. \\
& \left.\quad+2\left(v_{\nu}+w_{\nu}\right)^{\frac{n+2}{n-2}} w_{\nu}-\frac{n-2}{n}\left(v_{\nu}+w_{\nu}\right)^{\frac{2 n}{n-2}}\right) d v o l_{S^{n}} .
\end{aligned}
$$

This implies

$$
\begin{aligned}
& \int_{S^{n}}\left(\frac{4(n-1)}{n-2}\left|d u_{\nu}\right|_{S^{n}}^{2}+n(n-1) u_{\nu}^{2}\right) d v o l_{S^{n}} \\
& \leq r_{\infty}-2 \int_{S^{n}}\left(\frac{4(n-1)}{n-2} \Delta_{S^{n}} u_{\nu}-n(n-1) u_{\nu}+r_{\infty} u_{\nu}^{\frac{n+2}{n-2}}\right) w_{\nu} d v o l_{S^{n}} \\
& -\int_{S^{n}}\left(\frac{4(n-1)}{n-2}\left|d w_{\nu}\right|_{S^{n}}^{2}+n(n-1) w_{\nu}^{2}-\frac{n+2}{n-2} r_{\infty} v_{\nu}^{\frac{4}{n-2}} w_{\nu}^{2}\right) d v o l_{S^{n}} \\
& +r_{\infty} \int_{S^{n}}\left(\frac{n-2}{n} v_{\nu}^{\frac{2 n}{n-2}}-\frac{n+2}{n-2} v_{\nu}^{\frac{4}{n-2}} w_{\nu}^{2}\right. \\
& \left.\quad+2\left(v_{\nu}+w_{\nu}\right)^{\frac{n+2}{n-2}} w_{\nu}-\frac{n-2}{n}\left(v_{\nu}+w_{\nu}\right)^{\frac{2 n}{n-2}}\right) d v o l_{S^{n}}
\end{aligned}
$$

by virtue of Corollary 4. By Proposition 5, we can find a positive constant $\eta$ such that

$$
\begin{aligned}
& \int_{S^{n}}\left(\frac{4(n-1)}{n-2}\left|d w_{\nu}\right|_{S^{n}}^{2}+n(n-1) w_{\nu}^{2}-\frac{n+2}{n-2} r_{\infty} v_{\nu}^{\frac{4}{n-2}} w_{\nu}^{2}\right) d v o l_{S^{n}} \\
& \geq \eta \int_{S^{n}}\left(\frac{4(n-1)}{n-2}\left|d w_{\nu}\right|_{S^{n}}^{2}+n(n-1) w_{\nu}^{2}\right) d v o l_{S^{n}} \\
& \geq \eta Y\left(S^{n}\right)\left(\int_{S^{n}} w_{\nu}^{\frac{2 n}{n-2}} d v l_{S^{n}}\right)^{\frac{n-2}{n}}
\end{aligned}
$$


if $\nu$ is sufficiently large. Using Hölder's inequality, we obtain

$$
\begin{gathered}
\int_{S^{n}}\left(\frac{4(n-1)}{n-2} \Delta_{S^{n}} u_{\nu}-n(n-1) u_{\nu}+r_{\infty} u_{\nu}^{\frac{n+2}{n-2}}\right) w_{\nu} d v o l_{S^{n}} \\
\geq-\left(\int_{S^{n}}\left|\frac{4(n-1)}{n-2} \Delta_{S^{n}} u_{\nu}-n(n-1) u_{\nu}+r_{\infty} u_{\nu}^{\frac{n+2}{n-2}}\right|^{\frac{2 n}{n+2}} d v o l_{S^{n}}\right)^{\frac{n+2}{2 n}} \\
\cdot\left(\int_{S^{n}}\left|w_{\nu}\right|^{\frac{2 n}{n-2}} d v o l_{S^{n}}\right)^{\frac{n-2}{2 n}}
\end{gathered}
$$

hence

$$
\begin{aligned}
& \int_{S^{n}}\left(\frac{4(n-1)}{n-2} \Delta_{S^{n}} u_{\nu}-n(n-1) u_{\nu}+r_{\infty} u_{\nu}^{\frac{n+2}{n-2}}\right) w_{\nu} d v o l_{S^{n}} \\
& \geq-\frac{1}{\eta Y\left(S^{n}\right)}\left(\int_{S^{n}}\left|\frac{4(n-1)}{n-2} \Delta_{S^{n}} u_{\nu}-n(n-1) u_{\nu}+r_{\infty} u_{\nu}^{\frac{n+2}{n-2}}\right|^{\frac{2 n}{n+2}} d v o l_{S^{n}}\right)^{\frac{n+2}{n}} \\
& -\frac{\eta Y\left(S^{n}\right)}{4}\left(\int_{S^{n}}\left|w_{\nu}\right|^{\frac{2 n}{n-2}} d v o l_{S^{n}}\right)^{\frac{n-2}{n}} .
\end{aligned}
$$

Moreover, we have

$$
\begin{aligned}
& \mid \int_{S^{n}}\left(\frac{n-2}{n} v_{\nu}^{\frac{2 n}{n-2}}-\frac{n+2}{n-2} v_{\nu}^{\frac{4}{n-2}} w_{\nu}^{2}\right. \\
& \left.\quad+2\left(v_{\nu}+w_{\nu}\right)^{\frac{n+2}{n-2}} w_{\nu}-\frac{n-2}{n}\left(v_{\nu}+w_{\nu}\right)^{\frac{2 n}{n-2}}\right) d v o l_{S^{n}} \mid \\
& \leq C \int_{S^{n}}\left|w_{\nu}\right|^{\frac{2 n}{n-2}} d v o l_{S^{n}} \\
& +C\left(\int_{S^{n}}\left|w_{\nu}\right|^{\frac{2 n}{n-2}} d v o l_{S^{n}}\right)^{\frac{n-2}{n} \min \left\{\frac{n}{n-2}, \frac{3}{2}\right\}} .
\end{aligned}
$$

Since $\left\|w_{\nu}\right\|_{H^{1}\left(S^{n}\right)} \rightarrow 0$ as $\nu \rightarrow \infty$, this implies

$$
\begin{aligned}
& r_{\infty} \int_{S^{n}}\left(\frac{n-2}{n} v_{\nu}^{\frac{2 n}{n-2}}-\frac{n+2}{n-2} v_{\nu}^{\frac{4}{n-2}} w_{\nu}^{2}\right. \\
& \left.\quad+2\left(v_{\nu}+w_{\nu}\right)^{\frac{n+2}{n-2}} w_{\nu}-\frac{n-2}{n}\left(v_{\nu}+w_{\nu}\right)^{\frac{2 n}{n-2}}\right) d v o l_{S^{n}} \\
& \leq \frac{\eta Y\left(S^{n}\right)}{2}\left(\int_{S^{n}}\left|w_{\nu}\right|^{\frac{2 n}{n-2}} d v o l_{S^{n}}\right)^{\frac{n-2}{n}}
\end{aligned}
$$

if $\nu$ is sufficiently large. Putting these facts together, the assertion follows. 


\section{Exponential CONVERGENCE}

In this section, we prove that the metric $g(t)$ converges exponentially to a metric of constant sectional curvature as $t \rightarrow \infty$. The following proposition plays a key role in our analysis.

Proposition 7. We can find positive real numbers $C$ and $t_{0}$, depending only on the initial data, such that

$$
r_{g(t)} \leq r_{\infty}+C\left(\int_{S^{n}}\left|R_{g(t)}-r_{\infty}\right|^{\frac{2 n}{n+2}} d v o l_{g(t)}\right)^{\frac{n+2}{n}}
$$

for all $t \geq t_{0}$.

Proof. Suppose this is not true. Then we can find a sequence of times $\left\{t_{\nu}: \nu \in\right.$ $\mathbb{N}\}$ such that $t_{\nu} \geq \nu$ and

$$
r_{g\left(t_{\nu}\right)}>r_{\infty}+\nu\left(\int_{S^{n}}\left|R_{g\left(t_{\nu}\right)}-r_{\infty}\right|^{\frac{2 n}{n+2}} d v o l_{g\left(t_{\nu}\right)}\right)^{\frac{n+2}{n}}
$$

for all $\nu \in \mathbb{N}$. By Proposition 6, there exists an infinite subset $I \subset \mathbb{N}$ and a positive constant $\eta$ such that

$$
\begin{aligned}
& \int_{S^{n}}\left(\frac{4(n-1)}{n-2}\left|d u\left(t_{\nu}\right)\right|_{S^{n}}^{2}+n(n-1) u\left(t_{\nu}\right)^{2}\right) d v o l_{S^{n}} \\
& \leq r_{\infty}+\frac{2}{\eta Y\left(S^{n}\right)}\left(\int_{S^{n}} \mid \frac{4(n-1)}{n-2} \Delta_{S^{n}} u\left(t_{\nu}\right)\right. \\
& \left.\quad-n(n-1) u\left(t_{\nu}\right)+\left.r_{\infty} u\left(t_{\nu}\right)^{\frac{n+2}{n-2}}\right|^{\frac{2 n}{n+2}} d v o l_{S^{n}}\right)^{\frac{n+2}{n}}
\end{aligned}
$$

for all $\nu \in I$. Using the transformation law of the scalar curvature under a conformal change of the metric, we obtain

$$
r_{g\left(t_{\nu}\right)} \leq r_{\infty}+\frac{2}{\eta Y\left(S^{n}\right)}\left(\int_{S^{n}}\left|R_{g\left(t_{\nu}\right)}-r_{\infty}\right|^{\frac{2 n}{n+2}} d \operatorname{vol}_{g\left(t_{\nu}\right)}\right)^{\frac{n+2}{n}}
$$

for all $\nu \in I$. This is a contradiction.

Corollary 8. We can find positive real numbers $C$ and $t_{0}$, depending only on the initial data, such that

$$
r_{g(t)} \leq r_{\infty}+C \int_{S^{n}}\left(R_{g(t)}-r_{g(t)}\right)^{2} d v o l_{g(t)}
$$

for all $t \geq t_{0}$. 
Proof. By Proposition 7, we have

$$
r_{g(t)} \leq r_{\infty}+C\left(\int_{S^{n}}\left|R_{g(t)}-r_{\infty}\right|^{\frac{2 n}{n+2}} d v o l_{g(t)}\right)^{\frac{n+2}{n}}
$$

for $t \geq t_{0}$. This implies

$$
\begin{aligned}
r_{g(t)} & \leq r_{\infty}+2 C\left(\int_{S^{n}}\left|R_{g(t)}-r_{g(t)}\right|^{\frac{2 n}{n+2}} d v o l_{g(t)}\right)^{\frac{n+2}{n}} \\
& +2 C\left(r_{g(t)}-r_{\infty}\right)^{2}
\end{aligned}
$$

for $t \geq t_{0}$. Thus, we conclude that

$$
r_{g(t)} \leq r_{\infty}+4 C\left(\int_{S^{n}}\left|R_{g(t)}-r_{g(t)}\right|^{\frac{2 n}{n+2}} d v o l_{g(t)}\right)^{\frac{n+2}{n}}
$$

if $t$ is sufficiently large. Hence, the assertion follows from Hölder's inequality.

Proposition 9. There exist positive real numbers $C$ and $\delta$, depending only on the initial data, such that

$$
\int_{T}^{\infty}\left(\int_{S^{n}}\left(R_{g(t)}-r_{g(t)}\right)^{2} d v o l_{g(t)}\right)^{\frac{1}{2}} d t \leq C e^{-\delta T}
$$

for all $t \geq 0$.

Proof. Using the identity

$$
\frac{d}{d t} r_{g(t)}=-\frac{n-2}{2} \int_{S^{n}}\left(R_{g(t)}-r_{g(t)}\right)^{2} d v o l_{g(t)}
$$

and Corollary 8, we obtain

$$
r_{g(t)}-r_{\infty} \leq C e^{-2 \delta t}
$$

for all $t \geq 0$. (Here, $C$ and $\delta$ are positive real numbers which depend only on the initial data.) This implies

$$
\begin{aligned}
& \int_{T}^{T+1}\left(\int_{S^{n}}\left(R_{g(t)}-r_{g(t)}\right)^{2} d v o l_{g(t)}\right)^{\frac{1}{2}} d t \\
& \leq\left(\int_{T}^{T+1} \int_{S^{n}}\left(R_{g(t)}-r_{g(t)}\right)^{2} d v o l_{g(t)} d t\right)^{\frac{1}{2}} \\
& \leq\left(\frac{2}{n-2}\left(r_{g(T)}-r_{g(T+1)}\right)\right)^{\frac{1}{2}} \\
& \leq\left(\frac{2}{n-2}\left(r_{g(T)}-r_{\infty}\right)\right)^{\frac{1}{2}} \\
& \leq C e^{-\delta T}
\end{aligned}
$$


for all $T \geq 0$. From this the assertion follows.

Proposition 9 is very useful because it rules out concentration of volume. In light of the results of Schwetlick and Struwe, this implies that the function $u(t)$ is uniformly bounded from above. Moreover, it is easy to see that the scalar curvature $R_{g(t)}$ is uniformly bounded from below. Hence, the Harnack inequality for linear elliptic equations (see [6], Theorem 8.18 on p. 194) implies that $u(t)$ is uniformly bounded away from 0 . Finally, Proposition 9 implies that the function $u(t)$ converges exponentially in $L^{2}\left(S^{n}\right)$. The limiting metric is smooth and has constant scalar curvature. Therefore, the limiting metric must have constant sectional curvature by Obata's theorem (see [9], Proposition 6.1).

\section{REFERENCES}

[1] A. Bahri, Critical points at infinity in some variational problems, Pitman Research Notes in Mathematics Series, 182. Longman Scientific \& Technical (1989)

[2] A. Bahri and J.M. Coron, The scalar curvature problem on the standard three-dimensional sphere, J. Funct. Anal. 95, 106-172 (1991)

[3] R. Benedetti and C. Petronio, Lectures on Hyperbolic Geometry, Springer Verlag (1992)

[4] S. Brendle, Convergence of the Yamabe flow for arbitrary initial energy, J. Diff. Geom. 69, 217-278 (2005)

[5] B. Chow, The Yamabe flow on locally conformally flat manifolds with positive Ricci curvature, Comm. Pure Appl. Math. 45, 1003-1014 (1992)

[6] D. Gilbarg and N. Trudinger, Elliptic Partial Differential Equations of Second Order, Springer-Verlag (2001)

[7] R.S. Hamilton, Lectures on geometric flows, 1989 (unpublished)

[8] J.M. Lee and T.H. Parker, The Yamabe problem, Bull. Amer. Math. Soc. 17, 37-91 (1987)

[9] M. Obata, The conjectures on conformal transformations of Riemannian manifolds, J. Diff. Geom. 6, 247-258 (1971)

[10] R.M. Schoen, Conformal deformation of a Riemannian metric to constant scalar curvature, J. Diff. Geom. 20, 479-495 (1984)

[11] H. Schwetlick and M. Struwe, Convergence of the Yamabe flow for large energies, J. Reine Angew. Math. 562, 59-100 (2003)

[12] L. Simon, Asymptotics for a class of non-linear evolution equations with applications to geometric problems, Ann. of Math. 118, 525-571 (1983)

[13] M. Struwe, A global compactness result for elliptic boundary value problems involving limiting nonlinearities, Math. Z. 187, 511-517 (1984)

[14] R. Ye, Global existence and convergence of the Yamabe flow, J. Diff. Geom. 39, 35-50 (1994)

Simon Brendle

Department of Mathematics

Stanford University

E-mail: brendle@math.stanford.edu 